\title{
Molecular tools for studying the major malaria vector Anopheles funestus: improving the utility of the genome using a comparative poly $(\mathrm{A})$ and Ribo-Zero RNAseq analysis
}

\author{
Gareth D. Weedall ${ }^{1 *}$, Helen Irving ${ }^{1}$, Margaret A. Hughes $^{2}$ and Charles S. Wondji ${ }^{1 *}$
}

\begin{abstract}
Background: Next-generation sequencing (NGS) offers great opportunities for studying the biology of insect vectors of disease. Prerequisites for successful analyses include high quality annotated genome assemblies and that techniques designed for use with model organisms be tested and optimised for use with these insects. We aimed to test and improve genomic tools for studying the major malaria vector Anopheles funestus.

Results: To guide future RNAseq transcriptomic studies of An. funestus, we compared two methods for enrichment of non-ribosomal RNA for analysis: enrichment of polyadenylated RNA and ribosomal RNA depletion using a kit designed to deplete human/rat/mouse rRNA. We found large differences between the two methods in the resulting transcriptomes, some of which is due to differential representation of polyadenylated and non-polyadenylated transcripts. We used the RNAseq data for validation and targeted manual editing of the draft An. funestus genome annotation, validating $62 \%$ of annotated introns, manually improving the annotation of seven gene families involved in the detoxification of xenobiotics and integrated two published transcriptomic datasets with the recently published genome assembly.

Conclusions: The mRNA enrichment method makes a substantial, replicable difference to the transcriptome composition, at least partly due to the representation of non-polyadenylated transcripts in the final transcriptome. Therefore, great care should be taken in comparing gene expression data among studies. Ribosomal RNA depletion of total RNA using a kit designed to deplete human/rat/mouse rRNA works in mosquitoes and, we argue, results in a truer representation of the transcriptome than poly(A) selection. The An. funestus genome annotation can be considerably improved with the help of these new RNAseq data and further guided manual gene editing efforts will be of great benefit to the Anopheles research community for studies of this insect's genome and transcriptome.
\end{abstract}

Keywords: Anopheles funestus, Mosquitoes, Transcriptomics, RNAseq, Genome annotation

\footnotetext{
* Correspondence: gareth.weedall@|stmed.ac.uk; charles.wondji@|stmed.ac.uk

${ }^{1}$ Vector Biology Department, Liverpool School of Tropical Medicine,

Pembroke Place, Liverpool L3 5QA, UK

Full list of author information is available at the end of the article
} 


\section{Background}

Anopheles funestus is a major vector species of malaria in sub-Saharan Africa [1]. Similar to the more extensively studied vector species Anopheles gambiae, resistance to commonly used insecticides is a growing problem in $A n$. funestus [2-9]. Efforts to improve the design and implementation of resistance management strategies require a good understanding of the molecular basis of resistance. Use of genomic tools is paramount to achieve this goal. In contrast to An. gambiae, for which significant progress has been made in its genomics since its genome was sequenced more than a decade ago [10], An. funestus has received much less attention. However, progress is being made as the genome of Anopheles funestus was recently sequenced as part of a program to sequence a number of Anopheles species genomes [11]. The draft genome assembly, in the form of unplaced scaffolds not assembled into chromosome sequences, is publicly available via the VectorBase web resource $[12,13]$. The draft genome assembly has been annotated, using the genome annotation pipeline MAKER [14, 15]. However, this annotation has not been extensively manually curated and is inaccurate for a number of known genes of interest such as cytochrome P450 monooxygenase and glutathione S-trasferase (GST) genes. Thus, more efforts are currently needed to maximise the usefulness of the $A n$. funestus genome and allow more studies to be performed such as transcriptome profiling or genome-wide association studies. One approach to optimise the quality of this genome is to use RNAseq to improve genome annotation.

RNAseq is a powerful method to study the transcriptomes of organisms, providing a rich dataset allowing transcriptional profiling as well as the identification of novel transcripts, alternative splicing and detection of expressed sequence polymorphisms $[16,17]$. However in the case of Anopheles mosquitoes, limited information have been generated to determine the best methodology for library preparation to ensure an accurate profiling of transcriptomes, notably between the enrichment of polyadenylated (poly(A)) RNA transcripts and the alternative method of ribosomal RNA (rRNA) depletion. Methods of mRNA enrichment have been shown to have an effect on the observed transcriptome [18-21]. Comparisons of poly(A) mRNA enrichment with rRNA depletion methods report that the depletion methods result in more reads aligned outside of annotated gene coding regions [18, 20]. This may be due to the presence of novel transcripts and/or a greater proportion of genomic DNA in the library. Also, the greater number of reads aligned within introns in depletion libraries could be derived from immature transcripts [21]. Depletion libraries show more even read distribution less 3' bias than poly-A libraries [18].

In malaria-transmitting mosquitoes, insecticide resistance is often due to 'metabolic resistance': up-regulation of genes involved in detoxification, such as cytochrome P450 and GST genes. This is particularly the case in An. funestus, for which pyrethroid resistance is caused by metabolic resistance and not by target site resistance, such as mutations in the voltage gated sodium channel gene. As there are no known genomic biomarkers to detect metabolic resistance, transcriptomic analyses are necessary. These analyses have commonly used microarrays to detect up-regulated genes [4-6, 8]. RNAseq may offer advantages over microarrays in that unannotated, or poorly annotated, transcripts can be defined and corrected using the sequence alignment, which is more data-rich than the results of microarray analysis. This data richness includes information on alternative splicing, as well as non-protein-coding RNA transcripts (ncRNA) that may play a role in differential gene expression.

Here, we perform an RNAseq experiment on Anopheles funestus (FANG strain), primarily to compare two methods to enrich messenger RNA (mRNA) relative to ribosomal RNA (rRNA): ribosomal depletion and poly(A) selection. We compare the results of both methods applied to total RNA from the same individual mosquitoes and identify the most differentially represented transcripts between the methods to identify non-polyadenylated transcripts. We use the RNAseq data along with published de novo assemblies of Anopheles funestus transcripts [22, 23] to improve the genome annotation for selected genes, focusing on detoxification associated gene families.

\section{Results and discussion}

\section{Preparation of transcriptome sequencing libraries}

Total RNA was extracted from pools of whole mosquitoes of the An. funestus FANG colony. Extracts showed a pink colouration, probably due to eye pigments. It was not known if this would affect the sequencing library quality, so one sample was split and bead clean-up carried out on one of the sub-samples, to compare results with its uncleaned counterpart and assess whether this discolouration had any effect on the sequencing results. BioAnalyzer traces of total RNA (Additional file 1) showed evidence of the 'hidden break' reported in other insect total RNA samples [24]: two peaks of approximately 2000 bp representing 18S rRNA and the 28S rRNA subunits alpha and beta. The total RNA traces also showed some 'spikiness' the cause of which was unclear. The profile of degraded RNA tends to look smoother, with a broad peak extending left of the major $18 \mathrm{~S} / 28 \mathrm{~S}$ rRNA peaks. Whether this spikiness would affect the sequencing results was not known at this stage.

After Ribo-Zero rRNA depletion or poly(A) mRNA enrichment, BioAnalyzer traces suggested that all samples were of sufficient quality for RNAseq library preparation, with no obvious quality differences among samples within each method (Additional file 1). The amount of rRNA 
remaining in the samples appeared to be much lower for Ribo-Zero depleted samples than for poly(A) enriched samples, where even after 3 rounds of poly(A) selection, samples displayed a large peak near 2000 bp (Additional file 1). Later analysis indicated that this peak may represent mitochondrial 16S rRNA.

Initial quality checking of the sequence read data showed that the 8 libraries were evenly represented in the sequenced pool (median 61,229,870 reads per library; range $44,384,316$ to $68,640,804$ reads) and of good quality, with little read trimming due to sequenced adapters or low quality base calls (full details in Additional file 1).

\section{Alignment of sequence reads to reference genome}

All read libraries were aligned to a reference consisting of 1392 scaffolds derived from the Anopheles funestus FUMOZ colony with the An. funestus mitochondrial genome sequence added. Broad alignment metrics are shown in Table 1. Overall, a median of 35,309,124 (range $21,002,025$ to $38,878,773$ ), or $57 \%$ (range $35 \%$ to $59 \%$ ) of the reads to be aligned could be aligned. Alignment of spliced reads is more difficult than for unspliced reads and leads to lower proportions of the starting libraries being aligned, but this alone is unlikely to account for the relatively low alignment rate. The incompleteness of the reference genome sequence and high levels of sequence polymorphism and genetic divergence between laboratory colonies FANG (derived from Angola) and FUMOZ (from Mozambique, used for sequencing the reference genome) may also contribute to it. A greater proportion of the poly(A) than the Ribo-Zero libraries tended to be aligned (mean difference $11 \%$, median $8 \%$ ). For the sample where an initial bead clean-up was applied ("F1_XP"), the proportion of reads aligned for the RiboZero library ("F1_XP_RZ"; $55 \%$ ) was greater than for the uncleaned sample ("F1_RZ"; $35 \%$ ). This difference was not seen for poly(A) libraries (59 \% for both "F1_XP_PA" and "F1_PA"). This suggests that the purity of the sample may affect the efficacy of the rRNA depletion reaction, which is supported by the ribosomal RNA mapping results shown in Table 2.

\section{Tag counting for annotated genomic features and analysis of the ribosomal RNA content of libraries}

Tag counting means quantifying read pairs or singletons (representatives of a single fragment of transcript) aligned in genomic regions annotated as genome features (e.g. protein coding genes or ncRNAs). As the RNAseq protocol used is strand-specific, tags can be aligned sense or antisense to a feature, which mean different things: i.e. that antisense tags come from an antisense transcript, rather than from the primary annotated transcript. Sense and antisense tag counting was performed for each sequence alignment.

To assess how much ribosomal RNA was represented in each sequence library (i.e. the success of the mRNA enrichment procedure) gene features annotated as rRNAs were extracted from the genome annotation. There were ten of these features (Table 2): 8 resulting from automated annotation of the genome assembly and 2 annotated on the mitochondrial genome. For putative nuclear $28 \mathrm{~S}$ and $18 \mathrm{~S}$ rRNA genes, poly(A) mRNA enrichment almost always (except for the F1_XP sample pair) resulted in fewer tags from these genes than Ribo-Zero rRNA depletion. Putative 5.8S rRNA genes rarely showed any tags (possibly due to ambiguous read alignment arising from nonuniqueness among multiple $5.8 \mathrm{~S}$ rRNA copies in the reference genome or to their small size). The mitochondrial $12 \mathrm{~S}$ rRNA gene showed the same trend as for nuclear $28 \mathrm{~S}$ and $18 \mathrm{~S}$ rRNA genes. However, the mitochondrial $16 \mathrm{~S}$ rRNA gene showed the opposite trend, with more tags in the poly(A) mRNA enriched libraries than in the Ribo-Zero depleted libraries.

Tag counting is summarised in Table 3. As counting used annotated genomic features, antisense counts should be much lower than sense counts. This was the case, with

Table 1 Metrics describing the transcriptome alignments

\begin{tabular}{llllllll}
\hline Sample ID & $\begin{array}{l}\text { Reads to align } \\
(\mathrm{R} 1+\mathrm{R} 2)\end{array}$ & Aligned reads (\%) $^{\mathrm{a}}$ & Aligned R1 (\%) $^{\mathrm{b}}$ & Aligned R2 (\%) $^{\mathrm{b}}$ & Aligned in pair (\%) $^{\mathrm{b}}$ & Properly paired (\%) $)^{\mathrm{b}, \mathrm{c}}$ & Singleton (\%) \\
\hline F1_XP_RZ & $67,895,442$ & $37,480,868(55 \%)$ & $20,409,963(54 \%)$ & $17,070,905(46 \%)$ & $28,766,542(77 \%)$ & $26,655,176(71 \%)$ & $8,714,326(23 \%)$ \\
F1_XP_PA & $65,605,318$ & $38,878,773(59 \%)$ & $21,731,643(56 \%)$ & $17,147,130(44 \%)$ & $29,122,168(75 \%)$ & $27,271,540(70 \%)$ & $9,756,605(25 \%)$ \\
F1_RZ & $60,479,632$ & $21,002,025(35 \%)$ & $11,311,755(54 \%)$ & $9,690,270(46 \%)$ & $15,677,600(75 \%)$ & $14,192,682(68 \%)$ & $5,324,425(25 \%)$ \\
F1_PA & $63,555,450$ & $37,671,216(59 \%)$ & $20,881,499(55 \%)$ & $16,789,717(45 \%)$ & $28,690,598(76 \%)$ & $26,499,330(70 \%)$ & $8,980,618(24 \%)$ \\
F2_RZ & $43,733,908$ & $24,067,157(55 \%)$ & $13,121,886(55 \%)$ & $10,945,271(45 \%)$ & $18,560,162(77 \%)$ & $17,136,240(71 \%)$ & $5,506,995(23 \%)$ \\
F2_PA & $60,656,692$ & $35,610,912(59 \%)$ & $19,661,565(55 \%)$ & $15,949,347(45 \%)$ & $27,281,408(77 \%)$ & $25,371,888(71 \%)$ & $8,329,504(23 \%)$ \\
F3_RZ & $54,693,906$ & $25,673,135(47 \%)$ & $13,831,002(54 \%)$ & $11,842,133(46 \%)$ & $19,086,154(74 \%)$ & $17,431,254(68 \%)$ & $6,586,981(26 \%)$ \\
F3_PA & $60,211,066$ & $35,007,336(58 \%)$ & $19,424,414(55 \%)$ & $15,582,922(45 \%)$ & $26,507,306(76 \%)$ & $24,701,646(71 \%)$ & $8,500,030(24 \%)$ \\
\hline
\end{tabular}

${ }^{a} \%$ of reads to align

$\mathrm{b}_{\%}$ of aligned reads

'Properly paired means both read and its mate are mapped to opposing strands of the reference sequence, with 3 ' ends innermost and within the allowed distance from each other (mean $0 \mathrm{bp}$, standard deviation $100 \mathrm{bp}$ ) 
Table 2 Tag counts for ten putative rRNA genes

\begin{tabular}{|c|c|c|c|c|c|c|c|c|c|}
\hline GeneID & Description & $\begin{array}{l}\text { Tag count, } \\
\text { F1_XP_RZ }\end{array}$ & $\begin{array}{l}\text { Tag count, } \\
\text { F1_XP_PA }\end{array}$ & $\begin{array}{l}\text { Tag count, } \\
\text { F1_RZ }\end{array}$ & $\begin{array}{l}\text { Tag count, } \\
\text { F1_PA }\end{array}$ & $\begin{array}{l}\text { Tag count, } \\
\text { F2_RZ }\end{array}$ & $\begin{array}{l}\text { Tag count, } \\
\text { F2_PA }\end{array}$ & $\begin{array}{l}\text { Tag count, } \\
\text { F3_RZ }\end{array}$ & $\begin{array}{l}\text { Tag count, } \\
\text { F3_PA }\end{array}$ \\
\hline AFUN015486 & 285 & 20,426 & 10,311 & 120,345 & 16,664 & 19,706 & 28,057 & 95,593 & 20,651 \\
\hline AFUN015548 & $28 \mathrm{~S}$ & 47,771 & 21,402 & 154,805 & 31,516 & 48,525 & 54,305 & 145,807 & 41,643 \\
\hline AFUN015372 & 185 & 3,064 & 4,289 & 24,027 & 4,787 & 6,759 & 8,400 & 17,239 & 6,504 \\
\hline AFUN015629 & $5.8 \mathrm{~S}$ & 0 & 0 & 0 & 0 & 1 & 0 & 0 & 0 \\
\hline AFUN015410 & $5.8 \mathrm{~S}$ & 13 & 6 & 6 & 1 & 0 & 3 & 4 & 5 \\
\hline AFUN015687 & $5.8 \mathrm{~S}$ & 0 & 0 & 0 & 0 & 0 & 0 & 0 & 0 \\
\hline AFUN015706 & $5.8 \mathrm{~S}$ & 0 & 0 & 0 & 0 & 0 & 0 & 0 & 0 \\
\hline AFUN015471 & $5.8 S$ & 1 & 0 & 0 & 0 & 0 & 0 & 0 & 0 \\
\hline 16S-rRNA & $16 \mathrm{~S}$ (mito.) & 768,823 & $2,673,376$ & 402,555 & $2,351,745$ & 272,148 & $1,927,215$ & 491,163 & $2,382,882$ \\
\hline 12S-rRNA & $12 S$ (mito.) & 51,260 & 6,613 & 20,543 & 7,110 & 10,995 & 3,066 & 21,513 & 3,032 \\
\hline Total (\%) ${ }^{\mathrm{a}}$ & & $\begin{array}{l}891,358 \\
(3.86 \%)\end{array}$ & $\begin{array}{l}2,715,997 \\
(11.17 \%)\end{array}$ & $\begin{array}{l}722,281 \\
(5.49 \%)\end{array}$ & $\begin{array}{l}2,411,823 \\
(10.34 \%)\end{array}$ & $\begin{array}{l}358,134 \\
(2.42 \%)\end{array}$ & $\begin{array}{l}2,021,046 \\
(9.20 \%)\end{array}$ & $\begin{array}{l}771,319 \\
(4.78 \%)\end{array}$ & $\begin{array}{l}2,454,717 \\
(11.28 \%)\end{array}$ \\
\hline
\end{tabular}

${ }^{\mathrm{a}}$ Total rRNA gene tag counts as a \% of total aligned tags for each sample

a ten-fold greater assignment of tags to features in the sense orientation than antisense. The count data generated for each genomic feature is the data analysed in differential gene expression (DGE) analysis. Therefore, in general, the more sense tag-count data available, the greater the power of DGE analysis to detect differential gene expression. Overall, the poly(A) mRNA-enriched libraries produced more "on target" sense tags, as a proportion of all aligned tags (between 7.5 and $9.2 \%$ more than Ribo-Zero), as might be expected given enrichment on the poly(A) tails of mRNA transcripts (Table 3 and Fig. 1). However, after excluding tag counts from 10 putative rRNA genes, the differences between Ribo-Zero and poly(A) samples were less marked and for all samples (between 1.3 and $2.7 \%$ more on-target tags for poly(A) ). Overall, "on target" sense tag counts (excluding rRNA genes) comprised around 47.9 to $56.4 \%$ of the total aligned tags. This relatively low proportion probably reflects the fact that $5^{\prime}$ and $3^{\prime}$ un-translated regions (UTRs) are rarely predicted by automated annotation pipelines, so a large proportion of tags from mRNA transcripts are not counted as such when using gene models mostly comprising protein coding regions. Overall (excluding rRNA genes), our differential "gene expression" analysis would be based on between 6.6 and 13.4 million tags per sample.

\section{Comparison of poly(A) mRNA-enriched and Ribo-Zero rRNA-depleted samples}

Usually, the variation between samples within a sample group (within-group variation) is smaller than that between samples from different sample groups (overall variation) because the former consists of technical and biological variation only, while the latter also contains variation due to the treatment effect. When the treatment effect is the dominant term of variation, the sample groups can be clearly separated by statistical tools. If the treatment effect is weak compared to the technical and biological variation the samples will be difficult to discriminate based on the data. Here, the treatment effect is the difference between poly(A) and Ribo-Zero mRNA enrichment methods

Table 3 Summary of sense tag counting

\begin{tabular}{llllll}
\hline Sample & Total tags $^{\mathrm{a}}$ & $\begin{array}{l}\text { Assigned to features, } \\
\text { sense orientation }(\%)^{\mathrm{b}}\end{array}$ & $\begin{array}{l}\text { Assigned to non-rRNA features, } \\
\text { sense orientation }(\%)^{\mathrm{b}, \mathrm{c}}\end{array}$ & $\begin{array}{l}\text { Assigned to features, } \\
\text { antisense orientation }(\%)^{\mathrm{b}}\end{array}$ & $\begin{array}{l}\text { Assigned to non-rRNA features, } \\
\text { antisense orientation }(\%)^{\mathrm{b}, \mathrm{c}}\end{array}$ \\
\hline F1_XP_RZ & $23,097,597$ & $13,325,951(57.69 \%)$ & $12,434,593(53.84 \%)$ & $1,293,286(5.60 \%)$ & $1,286,090(5.57 \%)$ \\
F1_XP_PA & $24,317,689$ & $16,120,214(66.29 \%)$ & $13,404,217(55.12 \%)$ & $1,234,142(5.08 \%)$ & $1,216,317(5.00 \%)$ \\
F1_RZ & $13,163,225$ & $7,379,904(56.06 \%)$ & $6,657,623(50.58 \%)$ & $836,937(6.36 \%)$ & $814,750(6.19 \%)$ \\
F1_PA & $23,325,917$ & $14,816,364(63.52 \%)$ & $12,404,541(53.18 \%)$ & $1,355,508(5.81 \%)$ & $1,320,535(5.66 \%)$ \\
F2_RZ & $14,787,076$ & $8,421,844(56.95 \%)$ & $8,063,710(54.53 \%)$ & $1,006,847(6.81 \%)$ & $1,001,013(6.77 \%)$ \\
F2_PA & $21,970,208$ & $14,411,931(65.60 \%)$ & $12,390,885(56.40 \%)$ & $1,237,662(5.63 \%)$ & $1,218,099(5.54 \%)$ \\
F3_RZ & $16,130,058$ & $8,495,428(52.67 \%)$ & $7,724,109(47.89 \%)$ & $997,369(6.18 \%)$ & $984,961(6.11 \%)$ \\
F3_PA & $21,753,683$ & $13,447,922(61.82 \%)$ & $10,993,205(50.53 \%)$ & $1,126,921(5.18 \%)$ & $1,112,413(5.11 \%)$ \\
\hline
\end{tabular}

\footnotetext{
${ }^{\mathrm{a} A}$ tag is a read pair or a single, un-paired read
}

$\mathrm{b}_{\%}$ of "Total tags"

'Tags assigned to features after subtracting tags assigned to 10 putative rRNA features 


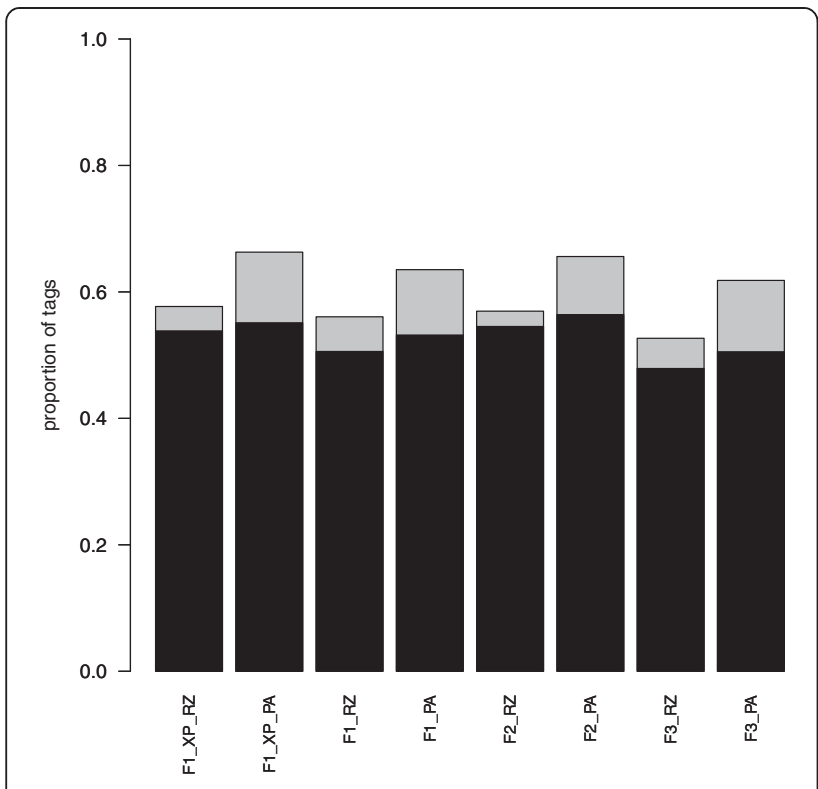

Fig. 1 Sense tag counts per annotated gene feature as a proportion of total aligned tags. Grey bars show total sense tag counts as a proportion of total aligned tags while black bars show this after excluding 10 putative rRNA genes

and a large treatment effect indicates that the mRNA enrichment/rRNA depletion method greatly affects the composition of the transcriptome. We assessed these effects.

Initial visual inspection of sequence alignments indicated that the Ribo-Zero libraries showed more 'background' alignments (reads aligned in intergenic and intronic regions) than the poly $(\mathrm{A})$ datasets. It is not certain whether this represents real, un-polyadenylated transcripts or possible erroneous sequencing of DNA. However, as samples came from the same pool and were DNase treated prior to being split, any difference in the amount of DNA being sequenced should be due to specific differences in the enrichment/depletion process.

As an initial gene-wise analysis of gene expression levels, reads or read pairs ("tags") mapped in the sense orientation to annotated gene models were counted for each sample. One caveat to this analysis is that the gene models used to generate tag counts were from a draft genome annotation and therefore were likely to contain errors. 257 of the 13,793 annotated features had sense tag counts of zero in all eight samples and were not analysed. To assess variation in the data, $\log _{2}$ sense tag counts per gene were plotted among sample pairs (Additional file 1). These give an indication of how similar are samples belonging to the same group and how different are samples from different sample groups. The associated correlation coefficients are displayed as a correlation heatmap (Additional file 1). Principal component analysis (PCA), using $\log _{2}$ sense tag counts per gene, was used to plot each sample relative to all others (Additional file 1). These analyses showed very low technical variation between the cleaned and uncleaned FANG-1 samples and greater biological variation among FANG-1, FANG-2 and FANG-3 samples. Ribo-Zero samples showed more biological variation than poly(A) samples, though this was mainly due to the FANG-2 RiboZero sample, which was a clear outlier (Additional file 1). FANG-2 Ribo-Zero was the smallest sample library, with around 44 million reads compared to around 55-62 million reads for the other samples, which may be associated with this result. Together, these assessments indicate that the mRNA enrichment method has a large effect on the sequenced transcriptomes of the samples.

\section{Analysis of differential transcriptome composition between mRNA enrichment methods}

To assess differences between the transcriptomes due to the mRNA enrichment method applied, differential gene expression (DGE) analysis was carried out to identify significantly differentially represented annotated genome features. First, as technical variation due to pre-enrichment sample cleaning of FANG-1 was low and to make full use of the data, the mean tag counts for cleaned and uncleaned samples were calculated and used in the DGE analysis. The data were modelled using a generalized linear model in a one-factor (mRNA enrichment method) experiment with two levels ("Ribo-Zero" and "poly(A)"). Full results of the analysis are shown in Additional file 2. Figure 2 shows the relationship between $\log _{2}$ fold change in expression between Ribo-Zero and poly(A) and mean expression level across all samples. The power in the data to discriminate between Ribo-Zero and poly(A) samples (clearly shown by PCA) is reflected in the large number of significantly differentially represented transcripts among the two transcriptomes. This analysis indicates that results based on Ribo-Zero and poly(A) mRNA enrichment may be very different from one another. Great care must therefore be taken when comparing results derived using the different methods.

In order to assess why the composition of Ribo-Zero and poly(A) transcriptomes differ so much, we looked at the 20 most differentially represented genome features in each group (ordered by FDR-adjusted $P$-value). Table 4 lists the 20 features most over- or under-represented in the Ribo-Zero transcriptome relative to poly(A). The two most over-represented features in the Ribo-Zero transcriptomes were ncRNAs, suggesting a large population of non-polyadenylated transcripts, including many ncRNAs, may exist in the cells and be under-represented in poly(A) transcriptomes. Two histone genes (histone H2A and H3) were over-represented in the Ribo-Zero transcriptome, consistent with the occurrence of replication-dependent, unpolyadenylated and replication-independent, polyadenylated histone forms $[19,25]$. Also, three genes with Cadherin domains were over-represented in the Ribo-Zero 


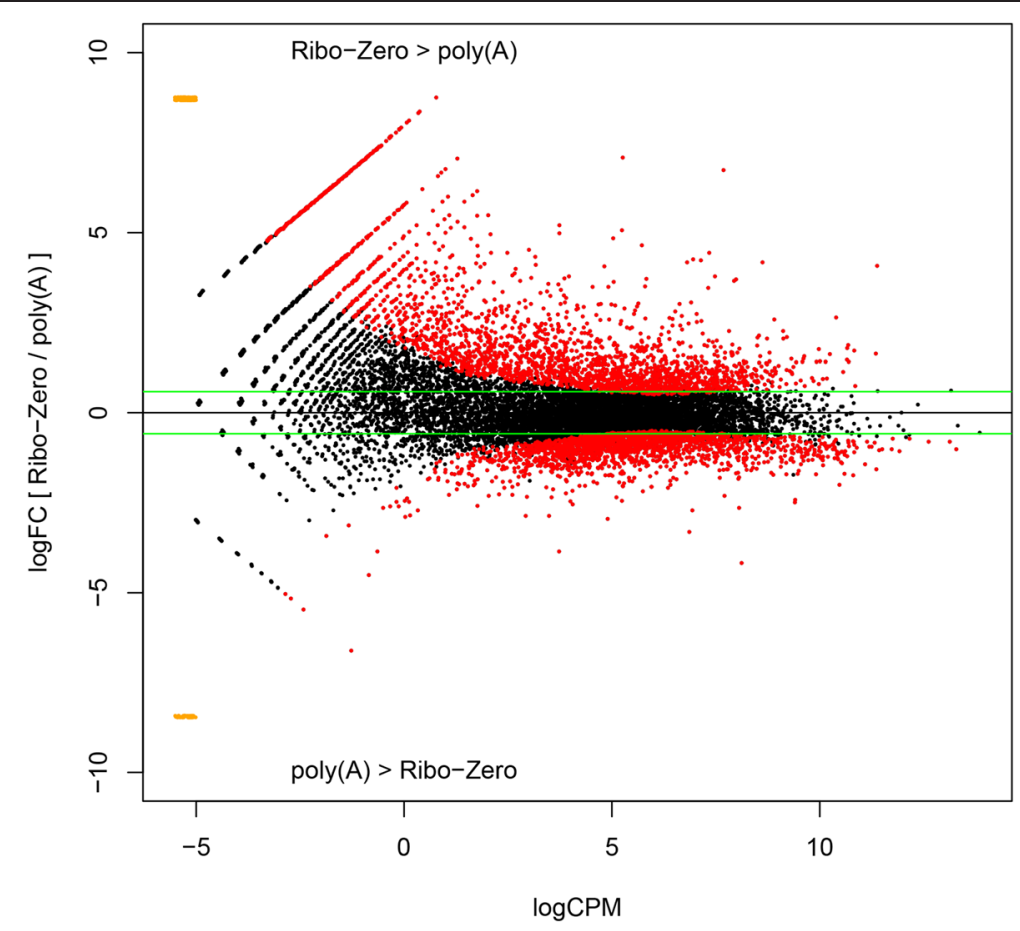

Fig. 2 Plot of differential transcript abundance in Ribo-Zero and poly(A) transcriptomes. The $y$-axis shows $\log _{2}$ fold-change of transcript abundance between Ribo-Zero and poly(A) samples. Greater than 0 indicates that a transcript is more abundant in Ribo-Zero samples than in poly(A) samples and vice versa, as indicated on the plot. Green lines indicate 1.5 -fold change. Red points are significantly differentially represented among treatments (FDR-adjusted $P$-values $<0.05$ ). Orange points are transcripts represented in only one sample, so fold-change could not be calculated. The $x$-axis shows the $\log _{2}$ average abundance of the transcripts in both Ribo-Zero and poly(A) transcriptomes. The shape of the plot and distribution of significantly differentially represented transcripts indicates that low abundance transcripts show more variation among Ribo-Zero and poly(A) transcriptomes due to sampling effects than do higher abundance transcripts

transcriptome. In other systems, Cadherin is among a set of genes controlled at the translational level by cytoplasmic polyadenylation [26]. If this were occurring in An. funestus, we would expect transcript forms with short poly(A) tails to be over-represented in the Ribo-Zero transcriptome. Further investigation would be needed to confirm if this is the case. Among the genes over-represented in the poly(A) transcriptome were a number of putative core metabolic genes such as transcription-associated proteins and ribosomal proteins. A study of poly(A) tail length in yeast showed that ribosomal proteins had long poly(A) tails [27]. We speculate that preferential enrichment of transcripts with longer poly(A) tails may affect the resulting transcriptome composition.

A broader analysis of functional categories and gene ontologies represented in the gene sets showed differences between poly(A) and Ribo-Zero libraries. The broad functional categories (as defined in the KEGG databases) of genes over-represented in the Ribo-Zero or poly(A) libraries were quite different. $28 \%(516 / 1,841)$ of genes were annotated for the Ribo-Zero set, compared to $51 \%$ $(643 / 1,250)$ for the poly(A) set and $36 \%(3702 / 10,253)$ for genes not differentially represented among treatments. The largest category (after "unclassified") for the
Ribo-Zero set was "environmental information processing", while for the poly(A) set it was "genetic information processing" (Fig. 3). Many of these "genetic information processing"-associated genes in the poly(A) set were ribosomal proteins, as indicated by gene ontology analysis (summarised in Table 5), where ribosomeassociated terms were enriched in this set (Fig. 4 and Additional file 1). In contrast, many of the "environmental information processing"-associated genes in the Ribo-Zero set may be membrane and/or nuclear proteins involved in signal transduction or transcriptional regulation (Fig. 4 and Additional file 1).

\section{Detection of un-annotated transcripts}

To further compare the transcriptomes, transcripts were annotated from aligned data from each method. 11,395 genes $(15,605$ transcripts) were predicted using the poly(A) data and 15,914 genes (20,317 transcripts) using the RiboZero data (Table 6). After removing transcripts that overlapped those predicted in the AfunF1.2 annotation, more remained in the Ribo-Zero transcriptome (nearly 5,000) that in the poly(A) transcriptome (around 1,000), suggesting the presence of transcripts not detected in the poly(A) transcriptome. Some of this difference could be due to an 
Table 4 The most significantly differentially represented genome features between Ribo-Zero and poly(A) transcriptomes

\begin{tabular}{|c|c|c|c|c|}
\hline Gene ID & $\operatorname{logFC}\left(\right.$ Ribo-Zero/poly(A)) ${ }^{\mathrm{a}}$ & $\log \mathrm{CPM}^{\mathrm{b}}$ & Adjusted $P$-value ${ }^{c}$ & Description \\
\hline AFUN015353 & 6.73 & 7.69 & $1.31 \mathrm{E}-89$ & Signal recognition particle (ncRNA) \\
\hline AFUN015615 & 7.08 & 5.27 & $2.65 E-75$ & Signal recognition particle (ncRNA) \\
\hline AFUN010049 & 5.05 & 5.25 & $3.80 \mathrm{E}-71$ & Histone H3 \\
\hline AFUN015157 & 4.18 & 6.81 & $5.86 E-62$ & - \\
\hline AFUN009381 & 4.64 & 5.73 & $7.90 \mathrm{E}-58$ & - \\
\hline AFUN008241 & 4.17 & 8.62 & $2.49 \mathrm{E}-47$ & - \\
\hline AFUN003688 & 4.43 & 7.35 & $1.91 \mathrm{E}-46$ & - \\
\hline AFUN014500 & 3.78 & 7.48 & $7.14 \mathrm{E}-44$ & - \\
\hline AFUN014369 & 3.71 & 5.84 & $1.51 \mathrm{E}-41$ & - \\
\hline AFUN015010 & 4.84 & 5.04 & $9.84 \mathrm{E}-41$ & - \\
\hline AFUN002493 & 3.46 & 7.32 & $3.88 \mathrm{E}-40$ & Unknown, contains cadherin domains \\
\hline AFUN000145 & 3.49 & 4.93 & $2.31 E-39$ & - \\
\hline AFUN008247 & 4.98 & 3.75 & $4.38 \mathrm{E}-37$ & - \\
\hline AFUN005891 & 5.19 & 3.75 & $7.85 \mathrm{E}-37$ & - \\
\hline AFUN011958 & 4.05 & 4.83 & $2.41 E-36$ & Retrotransposon, putative \\
\hline AFUN011227 & 3.50 & 5.80 & $1.43 \mathrm{E}-35$ & Unknown, contains cadherin domains \\
\hline AFUN008652 & 3.03 & 5.65 & $3.24 \mathrm{E}-34$ & Histone $\mathrm{H} 2 \mathrm{~A}$ \\
\hline AFUN011228 & 3.62 & 5.06 & $3.96 \mathrm{E}-33$ & Unknown, contains cadherin domains \\
\hline AFUN000984 & 2.81 & 6.53 & $2.55 \mathrm{E}-32$ & Unknown, contains PH-domain \\
\hline AFUN002265 & 2.94 & 5.61 & 4.67E-32 & odz/ten-m gene, putative \\
\hline AFUN010223 & -2.72 & 6.94 & $5.66 \mathrm{E}-26$ & $60 S$ ribosomal protein L39 \\
\hline AFUN004938 & -2.45 & 5.25 & $4.22 \mathrm{E}-25$ & Small nuclear ribonucleoprotein D3 \\
\hline nad3 & -4.18 & 8.12 & 1.15E-22 & $\mathrm{Nad} 3$ \\
\hline AFUN005575 & -2.96 & 4.91 & $1.03 \mathrm{E}-21$ & Estrogen receptor binding site associated antigen 9 variant 1 \\
\hline AFUN008128 & -2.06 & 5.96 & $9.59 \mathrm{E}-19$ & Transcription initiation factor TFIIF subunit alpha \\
\hline AFUN005032 & -1.86 & 6.56 & $6.57 \mathrm{E}-18$ & mRNA turnover protein 4 \\
\hline AFUN014198 & -2.51 & 9.41 & $1.94 \mathrm{E}-16$ & 40 S ribosomal protein $\$ 29$ \\
\hline AFUN011059 & -2.05 & 5.27 & $6.44 \mathrm{E}-15$ & - \\
\hline AFUN001216 & -1.94 & 5.18 & $1.12 \mathrm{E}-14$ & - \\
\hline AFUN006762 & -2.43 & 9.41 & 4.03E-14 & - \\
\hline AFUN009976 & -1.82 & 6.48 & $9.17 \mathrm{E}-14$ & Nuclear protein NHN1 \\
\hline AFUN014520 & -2.04 & 5.27 & $9.09 \mathrm{E}-13$ & CCR4-NOT transcription complex subunit $7 / 8$ \\
\hline AFUN014691 & -1.91 & 5.50 & 9.63E-13 & - \\
\hline AFUN002476 & -3.32 & 6.87 & $2.21 \mathrm{E}-12$ & - \\
\hline nad6 & -2.65 & 8.06 & $2.44 \mathrm{E}-12$ & Nad6 \\
\hline AFUN009128 & -1.69 & 5.29 & $2.86 \mathrm{E}-12$ & Nat13 protein \\
\hline AFUN007527 & -2.88 & 2.97 & $5.92 \mathrm{E}-12$ & Phosphopantothenoylcysteine decarboxylase \\
\hline AFUN005236 & -1.84 & 8.05 & $6.25 \mathrm{E}-12$ & 40 S ribosomal protein S21 \\
\hline AFUN003657 & -1.77 & 5.92 & $6.78 \mathrm{E}-12$ & - \\
\hline AFUN010447 & -2.04 & 4.37 & $1.08 \mathrm{E}-11$ & 26 proteasome complex subunit DSS1 \\
\hline
\end{tabular}

${ }^{a} \log _{2}$ fold change between 'expression' levels in Ribo-Zero and poly(A) samples (for values $>0$, Ribo-Zero $>$ poly $(A)$; for values $<0$, poly $(A)>$ Ribo-Zero)

${ }^{b} \mathrm{Log}_{2}$ counts per million mapped reads. Mean 'expression' level for all samples

'Significance of differential 'expression', $P$-value adjusted for a $5 \%$ false discovery rate 

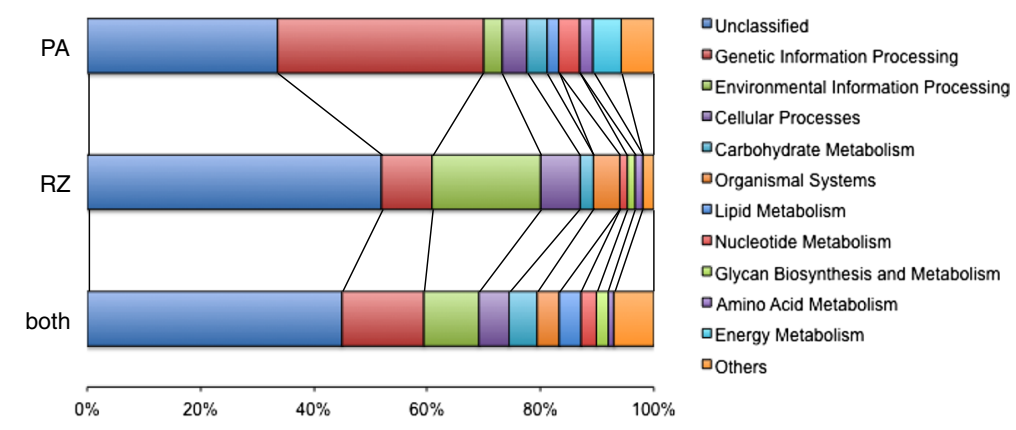

Fig. 3 Representation of functional categories in poly(A) and Ribo-Zero over-represented gene sets and in genes not differentially represented ("both")

increased amount of immature transcripts (that are neither fully spliced nor polyadenylated) and/or a greater contribution from DNA contamination in the Ribo-Zero samples (which are subjected to less PCR amplification specifically of transcripts). However, visual inspection of a sample of these putative transcripts showed that a number were indeed unannotated, Ribo-Zero-specific transcribed regions that did not occur within the introns of annotated genes and were predominantly strand-specific (i.e. did not result from DNA contamination, which would not be strandspecific). An example is shown in Fig. 5. Further analyses are needed to elucidate the functions of these transcripts.

\section{Validation and improvement of the annotation of Anopheles funestus genes, focused on detoxification- associated gene families}

An important part of genome annotation is validation of the predicted gene models. These RNAseq data allowed this to be done. On a genome-wide scale there were 44,031 predicted introns, of which 43,852 were unique (i.e. not the same intron in multiple transcript isoforms or overlapping genes). Of these, 28,346 (61.64 \%) were validated by at least 10 reads in the FANG-1, 2 and 3 poly(A) data sets $(31,927$ introns, $72.91 \%$, were validated by at least 1 read in the same data set). A small number of introns were supported at only one end, suggesting mis-annotation of the gene models. Of the 44,031 predicted introns, 1,125 were supported only at the $5^{\prime}$ end and 2,118 only at the 3 ' end. Introns supported only at the $3^{\prime}$ end tend to be the first predicted intron ( $45 \%$ the first intron vs. $13 \%$ the last intron), while introns supported only at the $5^{\prime}$ end tend to be the last predicted intron (33\% the last intron vs. $17 \%$ the first intron), suggesting that the ends of up to around 3,000 gene models are mis-annotated and could be manually improved. Results of the validation analysis for all predicted introns are shown in Additional file 3. For the 28,346 validated introns, consensus motifs at intron-exon boundaries are shown in Fig. 6. The 5' end of the intron has an extended GTAAGT motif, while the 3 ' end of the intron has the conserved AG motif preceded by an extended pyrimidine-rich region. No conserved motif is seen in the flanking exons, except for a slight enrichment of purines at the last position of the upstream exon.

Visual inspection of the automated MAKER genome annotation of Anopheles funestus showed that, in many cases, the MAKER annotation had inferred a single gene model from multiple closely located genes. This behaviour is particularly problematic for detoxification-associated genes such as cytochrome P450 and GST because these genes often occur in clusters of tandemly duplicated paralogous genes. Manual editing was required to improve the genome annotation, an example of which is shown in Fig. 7.

We aimed to improve the annotation of genes involved in the three phases of detoxification: transformation (phase I), conjugation (phase II) and transport/efflux (phase III). Two major enzyme families, P450 (phase I) and GST (phase I and II), have already been manually curated and, at the time of writing, are being incorporated into the VectorBase genome annotation, AfunF1.3 (Dr. Craig Wilding and Dr. Dan Lawson, personal communications). Therefore, we focused upon seven other detoxification gene families. These were: the phase I enzymes carboxylesterases (COE),

Table 5 Summary of gene ontology analysis

\begin{tabular}{|c|c|c|c|c|c|c|}
\hline GO domain & Instances $^{\mathrm{a}}\left(\mathrm{BG}^{\mathrm{b}}\right)$ & Instances $\left(R Z^{b}\right)$ & Instances $\left(\mathrm{PA}^{\mathrm{b}}\right)$ & RZ vs. $B^{c}{ }^{c}$ & PA vs. $\mathrm{BG}^{\mathrm{C}}$ & RZ vs. $P^{c}$ \\
\hline Biological process & 7,228 & 763 & 711 & 11 & 9 & 19 \\
\hline Molecular function & 14,362 & 1,912 & 1,109 & 15 & 13 & 25 \\
\hline Cellular compartment & 3,648 & 403 & 515 & 4 & 10 & 10 \\
\hline
\end{tabular}

aAn "instance" refers to a GO term associated with a gene ID

${ }^{\mathrm{b}} \mathrm{BG}=$ background (whole genome); $\mathrm{RZ}=$ Ribo-Zero; $\mathrm{PA}=\operatorname{poly}(\mathrm{A})$

'Significantly differentially represented GO terms (adjusted $p<0.05$ ) 


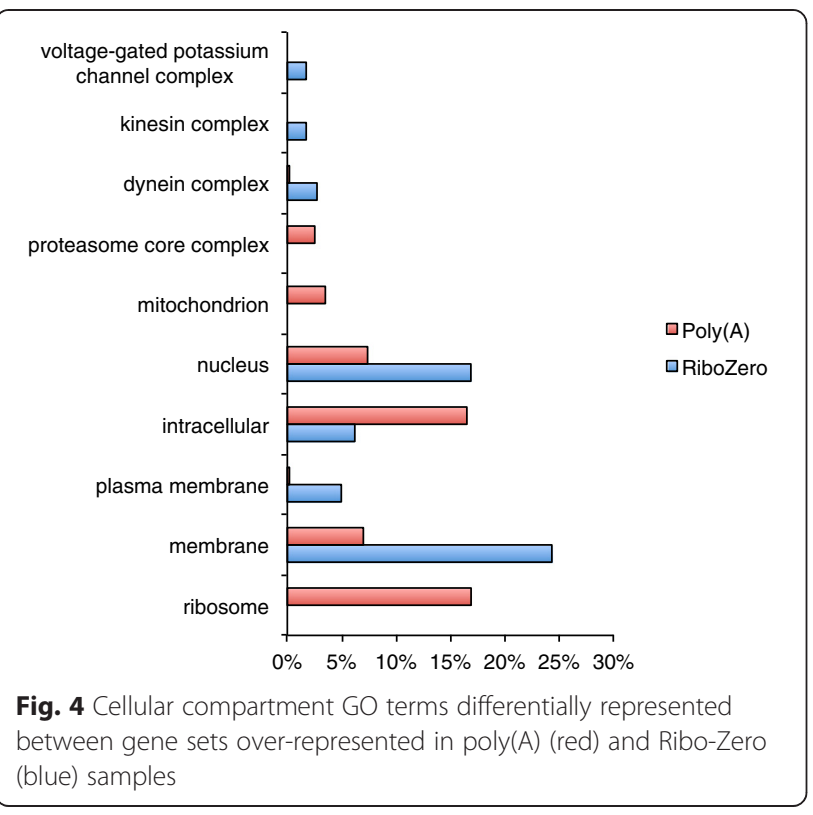

flavin-containing monooxygenases (FMO), aldehyde oxidases (AOX) and aldehyde dehydrogenases (ALDH); the phase II enzymes UDP glucosyltransferases (UGT) and sulfotransferases (SULT); and the phase III transporter-encoding ATP-binding cassette genes $(\mathrm{ABC})$.

Manual editing considerably improved the annotation of detoxification-associated genes. A table of manually edited gene models and unedited, putatively correct, gene models belonging to each family is shown in Additional file 4 .

For the carboxylestarase genes (COE), 22 gene models were manually edited to produce 28 putative COE genes and 6 non-COE genes, 10 gene models were not modified. For the flavin-containing monooxygenase genes (FMO) 4 gene models were identified and were not modified. For the aldehyde oxidase genes (AO), 9 gene models were manually edited to produce 6 putative AO genes, 2 xanthine dehydrogenase genes and 2 non-AO genes, 2 gene models were not modified. For the aldehyde dehydrogenase genes (ALDH), 2 gene models were manually edited to produce 2 ALDH genes and 1 non-ALDH gene, 1 gene model was not modified.

For the UDP-glycosyltransferase genes (UGT), 4 gene models were manually edited to produce 8 UGT genes

Table 6 Comparison of the published genome annotation with de novo annotations based on RNAseq alignments

\begin{tabular}{llll}
\hline & AfunF1.2 $^{\text {a }}$ & $\begin{array}{l}\text { Poly(A) (not } \\
\left.\text { in ref }^{\mathrm{b}}\right)\end{array}$ & $\begin{array}{l}\text { Ribo-Zero (not } \\
\left.\text { in ref }^{\mathrm{b}}\right)\end{array}$ \\
\hline $\begin{array}{l}\text { Scaffolds with annotation } \\
\text { Genes }\end{array}$ & 622 & $587(415)$ & $727(645)$ \\
Transcripts & 13,757 & $11,395(1,000)$ & $15,914(4,744)$ \\
\hline
\end{tabular}

${ }^{a}$ Excludes mitochondrial genome

${ }^{\mathrm{b}}$ Features that do not overlap any in the reference annotation and 3 non-UGT genes, 14 gene models were not modified. For the sulfotransferase genes (SULT), 1 gene model was manually edited to produce 5 SULT genes and and an oestrogen sulfotransferase gene, 3 gene models were not modified.

For the ATP-binding cassette genes (ABC), 9 gene models were manually edited to produce $13 \mathrm{ABC}$ genes and 10 non- $A B C$ genes, 16 gene models were not modified.

All manually edited gene models have been submitted to VectorBase for incorporation into the Anopheles funestus genome annotation.

\section{Integration of An. funestus transcriptome datasets and assignment of FUMOZ scaffolds to chromosome arms}

Two An. funestus whole transcriptome datasets were published before the whole genome assembly [22, 23]. We integrated both the "Crawford" transcriptome (14,850 sequences) and the "Gregory" transcriptome (18,103 sequences) with the FUMOZ predicted transcript set, AfunF1.2 (13,897 sequences) by clustering them based on sequence similarity. The results are shown in Additional file 5. Clustering the full set of 46,850 sequences at $95 \%$ similarity resulted in 25,519 clusters. The results suggested that the "Gregory" transcriptome was more fragmented than the other two: its 18,103 sequences were represented in only 5,741 unique clusters, compared to 12,349 clusters for the "Crawford" transcriptome and 13,172 clusters for the AfunF1.2 transcript set.

Genome sequences assembled to the level of whole chromosomes are more useful than draft assemblies for analyses of genome wide variation and gene expression, because structural and spatial information can be important (e.g. for defining the location of a selective sweep or the effect of regional chromatin remodelling on co-regulation of gene expression). The comparison of An. funestus predicted genes to those of An. gambiae allowed a number of scaffolds to be tentatively assigned to chromosome arms based on homology to An. gambiae. Table 7 summarises the number of scaffolds with 5 or more genes assigned to a chromosome arm by putative 1:1 orthology with An. gambiae (all scaffold IDs are listed in Additional file 6). In a small number of anomalous cases where a scaffold contained genes with orthology to genes on a different chromosome An. gambiae arm, the minority chromosome arm was never supported by more than 1 gene and these were removed by setting a threshold of 5 genes to support assignment of a scaffold to a group. This conservative approach to defining linkage groups assigned only a small number (286/ $1392=20.55 \%)$ of scaffolds. However, these were generally large scaffolds and represented the greater proportion $(183,517,537 / 225,223,604=81.48 \%)$ of the total genome length. These groups could form a starting 


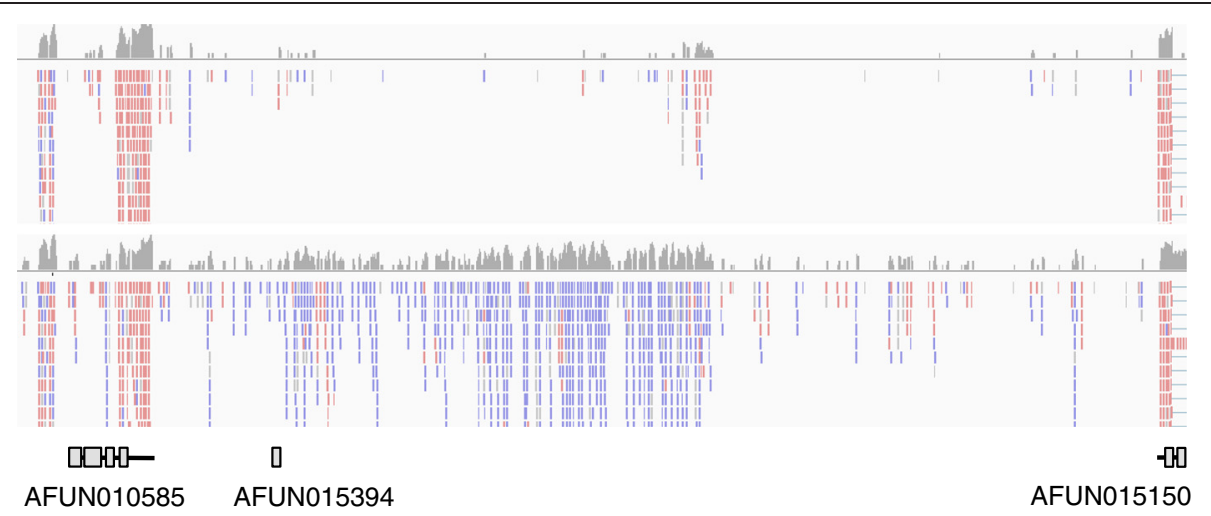

Fig. 5 Example of putative novel transcript(s) represented in the Ribo-Zero libraries but not in the poly(A) libraries. The top panel shows a coverage depth summary plot (grey plot; log-scaled coverage depth) and aligned reads (red bars are reads from pairs aligned to the plus strand, blue bars from pairs aligned to the minus strand) for the poly(A) data. Below these are the same for the Ribo-Zero data. Below these are gene models (grey boxes represent exons, black lines un-translated regions: introns and UTRs) from the gene set AfunF1.2. A large transcribed region on the minus strand is evident in the Ribo-Zero data only, representing one or more putatively poly(A)- transcripts

point for genome assembly finishing and for further analysis of synteny and co-linearity of genes between $A n$. funestus and An. gambiae.

\section{Conclusions}

The sequencing of the genome of the major malaria vector Anopheles funestus allows new analyses of its biology and may accelerate research on this species, which currently lags behind that of Anopheles gambiae. To carry out analyses of gene expression relevant to insecticide resistance, we have piloted the use of RNAseq. In addition, we have improved the genome annotation for a number of gene families with roles in the detoxification of xenobiotic agents.

As reported in other eukaryotic systems, the method used to enrich for mRNA relative to rRNA has a large effect upon the observed transcriptome [18-21]. Functional classes of genes are differentially represented in poly(A) mRNA-enriched and Ribo-Zero rRNA-depleted transcriptomes, with greater representation of ribosomal proteins with poly(A) and membrane-associated proteins with RiboZero. Genes with both poly $(\mathrm{A})+$ and poly $(\mathrm{A})$ - transcripts, such as histones, and ncRNA, such as signal recognition particle RNAs, were also highly represented in the RiboZero transcriptome. The Ribo-Zero libraries show a higher proportion of reads aligning outside of annotated exons, which may represent a combination of novel, poly(A)- transcripts and the introns of immature transcripts.

In making recommendations for which method to use for studies of the transcriptomes of mosquitoes, several factors should be considered. For analyses of differential gene expression, power to detect different expression levels is proportional to the number of reads aligned to each gene. Poly(A) mRNA enrichment does provide more of this 'on-target' data than Ribo-Zero rRNA depletion, yet the difference is less striking after accounting for rRNA genes, in particular a highly poly(A)-enriched mitochondrial 16S rRNA. The transcriptome profiles of poly(A) samples were more highly correlated with each other than Ribo-Zero samples, indicating that power to detect differential gene expression would be greater for poly(A) mRNA-enriched samples. However, we argue that Ribo-Zero rRNA depletion produces a truer representation of the transcriptome because (i) there is less

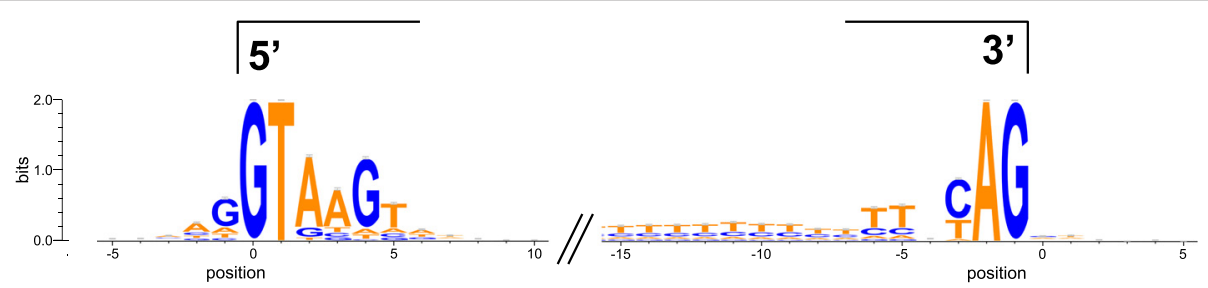

Fig. 6 Consensus sequences at the $5^{\prime}$ and $3^{\prime}$ intron-exon boundaries of 28,346 introns validated by RNAseq. Position 0 at the $5^{\prime}$ end marks the first nucleotide in the intron. Position 0 at the $3^{\prime}$ end marks the first nucleotide in the following exon. Two diagonal lines denote the variable central portions of the introns. The $y$-axis indicates the bit-score for each nucleotide position. An extended motif of GTAAGT is seen at the $5^{\prime}$ end, with a slight enrichment of purines at the last position of the preceding exon. The conserved AG motif is seen at the $3^{\prime}$ end, preceded by an extended pyrimidine-rich region. Little or no sequence conservation is seen in the flanking exons, apart from at the last position of the preceding exon 


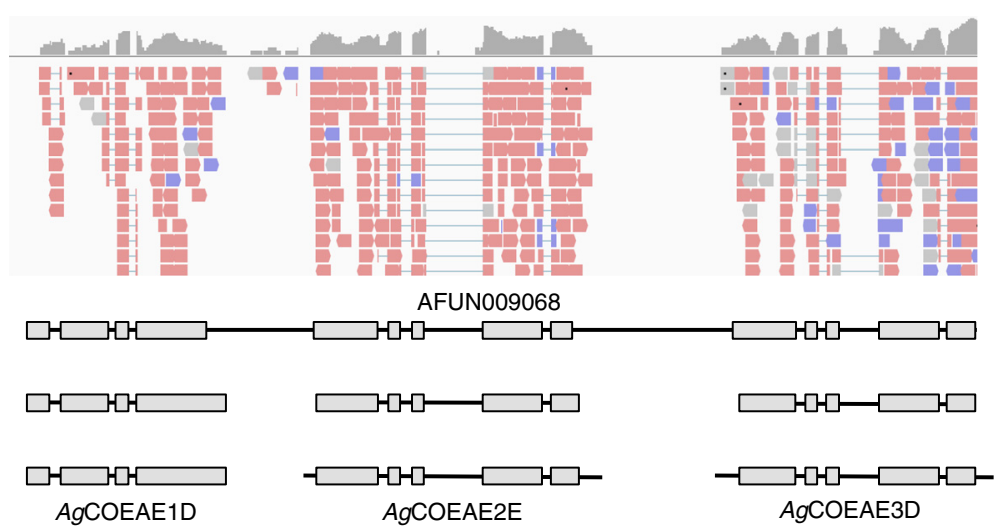

Fig. 7 Example of an incorrect gene model (AFUN009068) and three manually edited gene models. The top panel shows a coverage depth summary plot (grey plot; log-scaled coverage depth) and aligned reads (red bars are reads from pairs aligned to the plus strand, blue bars from pairs aligned to the minus strand). Below these are the gene models (grey boxes represent exons, black lines un-translated regions: introns and UTRs). The gene model in gene set AfunF1.2 (AFUN009068) is shown above three manually edited gene models based on read alignments and orthologous genes in the Anopheles gambiae $(\mathrm{Ag})$ genome annotation (bottom). The three genes are carboxylesterases (COE)

PCR amplification of transcripts, which can bias the final results and (ii) The transcriptome includes the poly(A)fraction, which may be functionally relevant. Due to the large effect that the mRNA enrichment method has on the transcriptome obtained, researchers should be very careful in comparing results obtained using different methods.

The draft genome annotation of An. funestus is currently inadequate for detailed studies of gene expression targeted at insecticide resistance mechanisms. Major gene families involved in detoxification of xenobiotic substances, such as cytochrome P450 and GST genes, are not well annotated by automated approaches. Manually edited gene models for these gene families are being incorporated into the genome annotation at the time of writing (Dr. Craig Wilding and Dr. Dan Lawson, personal communications). We extended this annotation improvement to 7 more key detoxification genes families: 4 associated with phase I; 2 with phase II and 1 with phase III. This effort offers a major improvement of the utility of the An. funestus genome as a tool to study

Table 7 Anopheles funestus scaffolds assigned to groups representing putative chromosome arms

\begin{tabular}{lll}
\hline${\text { Chromosome } \text { arm }^{a}}^{a}$ & Scaffolds $^{b}$ & Total length (bp) \\
\hline X & 27 & $16,915,574$ \\
2R & 78 & $52,607,791$ \\
2 L & 57 & $42,990,663$ \\
3R & 72 & $41,849,609$ \\
$3 \mathrm{~L}$ & 52 & $29,153,900$ \\
Total & 286 & $183,517,537$ \\
\hline
\end{tabular}

${ }^{a}$ An. funestus chromosome arm, accounting for the whole arm translocation between $2 \mathrm{~L}$ and $3 \mathrm{R}$ relative to An. gambiae

${ }^{\mathrm{b}}$ Number of An. funestus scaffolds with 5 or more putative 1:1 orthologues with An. gambiae

' Length includes sequencing gaps insecticide resistance in this species. Accurate annotation of these gene families is particularly important for An. funestus, as insecticide resistance appears to be primarily mediated by detoxification of the compounds in this species.

\section{Methods}

\section{Mosquito samples used in the study}

The study used Anopheles funestus mosquitoes of the FANG laboratory colony, a fully insecticide susceptible colony derived from Angola [28]. Mosquito eggs (a kind gift of Prof. Lizette Koekemoer, University of the Witwatersrand, South Africa) were hatched and mosquitoes reared to adulthood in the insectaries at the Liverpool School of Tropical Medicine under conditions described elsewhere [2].

\section{RNA extraction, sequence library preparation and sequencing}

Total RNA was extracted from three pools of 10 individual adult female mosquitoes using the Arcturus PicoPure RNA isolation kit (Life Technologies, Carlsbad, USA), according to the manufacturer's instructions and including a DNase treatment step. On visual inspection of the purified RNA, the samples displayed some pigmentation, the likely effect of which on data quality was unknown. To assess this, the most abundant sample (FANG-1) was split in two. One half of the sample was cleaned using Agencourt AMPure XP beads (Beckman Coulter, Brea, USA) prior to further processing.

All four samples were split in two and one of each pair subjected to poly(A) mRNA enrichment and the other to ribosomal RNA depletion. Polyadenylated RNA was selected from total RNA samples using 3 rounds of poly(A) selection with the Dynabeads mRNA purification kit (Life 
Technologies), using $1.5 \mu \mathrm{g}$ of starting material. Total RNA was rRNA-depleted using the Ribo-Zero low input kit for Human/Mouse/Rat (Illumina, San Diego, USA), using $100 \mathrm{ng}$ of starting material. RNAseq libraries were prepared from poly(A) and Ribo-Zero mRNA-enriched material using the ScriptSeq v2 RNAseq library preparation kit (Illumina), using 15 cycles of PCR amplification. Libraries were purified using Agencourt AMPure XP beads (Beckman Coulter). Each library was quantified using a Qubit fluorometer (Life Technologies) and the size distribution assessed using the 2100 Bioanalyzer (Agilent Technologies, Santa Clara, USA).

The eight final libraries were pooled in equimolar amounts using the Qubit and Bioanalyzer data. The quantity and quality of each pool was assessed by Bioanalyzer and subsequently by qPCR using the Kapa Illumina library quantification kit (Kapa Biosystems, Wilmington, USA), on a Light Cycler LC480II (Roche, Basel, Switzerland), according to manufacturers' instructions. The pool of libraries was sequenced on one lane of the HiSeq 2500 (Illumina) at $2 \times 125 \mathrm{bp}$ paired-end sequencing with $\mathrm{v} 4$ chemistry. Sequence library preparation and sequencing were done at the Centre for Genomic Research, University of Liverpool, UK.

\section{Alignment of sequence reads to a reference genome}

Initial processing and quality assessment of the sequence data was performed using a software pipeline developed at the Centre for Genomic Research, University of Liverpool (Dr. Richard Gregory, personal communication). In this pipeline, basecalling and de-multiplexing of indexed reads was performed by CASAVA version 1.8.2 (Illumina) to produce samples from the pooled sequence data, in fastq format. The raw fastq files were trimmed to remove Illumina adapter sequences using Cutadapt version 1.2.1 [29]. The 3' end of any read matching the adapter sequence over at least $3 \mathrm{bp}$ was trimmed off. Reads were further trimmed to remove low quality bases, using Sickle version 1.200 [30], with a minimum window quality score of 20. After trimming, reads shorter than $10 \mathrm{bp}$ were removed. If both reads from a pair passed this filter, each was included in either the R1 (forward reads) or R2 (reverse reads) file. If only one of a read pair passed this filter, it was included in the R0 (unpaired reads) file.

The reference sequence used for alignment was the Anopheles funestus assembled scaffold sequences derived from the FUMOZ laboratory colony, a multi-insecticide resistant colony derived from southern Mozambique $[11,28]$. Assembly AfunF1 (GenBank assembly identifier GCA_000349085.1; GenBank WGS project identifier APCI01) was downloaded from VectorBase [12, 13]. A single sequence representing the Anopheles funestus mitochondrial genome (accession number DQ146364)
[31] was added to these scaffolds to make the reference sequence used in the analysis.

R1/R2 read pairs were aligned to the reference sequence using TopHat version 2.0.10 [32], based on the bowtie 2 aligner [33]. The alignment was carried out using the following non-default parameters: the insert inner distance (between $3^{\prime}$ ends of R1 and R2 reads) was set to 0 , with a standard deviation of 50 (options "-mate-inner-dist 0" and "-mate-std-dev 50"); for reads with multiple alignments in the genome(s), only a single, best alignment was recorded, with ties assigned randomly (option "-max-multihits 1"); the library type was specified as second-stranded (option "-library-type frsecondstrand"), as it was a ScriptSeq library (in second strand sequencing, the forward read of a pair, R1, will map sense to a transcript, and the reverse read, R2, will map antisense).

\section{Analysis of transcript abundance among samples}

Tags mapped in the sense orientation to annotated Anopheles funestus genes (automated predictions from gene set AfunF1.2, 2014-08-22, downloaded from VectorBase and annotated genes from the mitochondrial genome) were counted using htseq-count, part of the 'HTSeq' framework, version 0.5.3p9 [34]. Read count data were analysed in the $\mathrm{R}$ environment, in particular using the package edgeR for differential gene expression analysis [35].

Analysis of the tag count data used $\log _{2}$ transformed counts (after replacing 0 values with $1 \mathrm{~s}$ ). Pairwise scatter plots of these values were plotted and the Pearson's correlation coefficients $\left(r^{2}\right)$ represented in a sample correlation heatmap. Principal component analysis (PCA) was applied and the first, second and third principal components of variation plotted.

Differential gene expression analysis was carried out using edgeR [35]. Due to the low level of technical variation between cleaned and uncleaned FANG-1 samples, differential gene expression analysis used the mean tag count values for cleaned and uncleaned FANG-1 samples for each enrichment method. Two groups of samples ("Ribo-Zero" and "poly(A)") were defined. Normalisation factors were calculated to correct for differences in total tag counts among samples, which may otherwise cause bias in differential gene expression analysis, using the "TMM" (Trimmed Mean M-values) method in edgeR [36] with default parameters. Common, trended (with a minimum of 500 genes in each bin) and tag-wise dispersion parameters were estimated. Trended dispersion was used for significance testing. Variation of RNAseq data can be modelled by a negative binomial distribution [37] and the data modelled using a generalized linear model [38]. For the contrast "Ribo-Zero / poly(A)", the estimated $\log _{2}$ Fold Change for each gene was tested in edgeR using a 
Likelihood-Ratios (LR) test [39]. P-values associated with $\log \mathrm{FC}$ were adjusted for multiple testing using the False Discovery Rate (FDR) approach [40]. Significantly differentially expressed genes were defined as those with an FDR-adjusted $P$-value $<5 \%$.

\section{Analysis of gene ontologies and KEGG functional categories}

All GO terms associated with features in genome annotation AfunF1.2 were obtained from VectorBase. The terms were separated by GO domain: biological process (BP; 7,228 instances), molecular function (MF; 14,362 instances) and cellular compartment (CC; 3,648 instances). An "instance" refers to a GO term associated with a gene ID and the term is used here because multiple GO terms can be associated with the same gene ID and multiple gene IDs with the same GO term. To test for significant differences in GO term representation among sets of genes, Fisher's exact test was applied for each GO term, with a correction for multiple testing using the method of Benjamini and Hochberg [40]. The gene sets tested were those over-represented in Ribo-Zero or in poly(A) libraries (with adjusted $p$-values $<0.01$ in the test for differential expression).

Proteins were annotated using blastKOALA v2.0 [41], a tool available via the Kyoto Encyclopedia of Genes and Genomes, KEGG [42-44]. Four sets of proteins were submitted to blastKOALA: those over-represented in Ribo-Zero libraries $(n=1841)$; those over-represented in poly(A) libraries $(n=1250)$; those not significantly differentially represented (two sets, as a maximum of 10,000 sequences can be submitted at a time: $n=5531$ and $n=4722$ ). The protein sequence files were uploaded and the taxonomy ID of An. funestus (taxid:62324) entered. The database searched against was the "genus_eukaryotes" database (containing $3,696,044$ entries).

\section{Detection of un-annotated transcripts}

Alignment files of the four poly(A) mRNA-enriched libraries and the four Ribo-Zero rRNA-depleted libraries (in bam format) were merged to create a single poly(A) and a single Ribo-Zero alignment. Alignments to the mitochondrial genome were filtered out. These filtered alignments were used to predict transcripts using Cufflinks v2.2.1 [45]. Default parameters were applied except for the options "-library-type" (set to "fr-secondstrand") and "-minfrags-per-transfrag" (set to 100 rather than the default 10). Putative transcripts were compared to the genome annotation using Cuffmerge and transcripts not associated with annotated features extracted for each of the poly(A) and the Ribo-Zero alignments. These transcripts were merged using Cuffmerge and the resulting file of features used in conjunction with the alignment files to generate read counts for poly(A) and the Ribo-Zero samples using featureCounts v1.4.6 [46].

\section{Genome annotation validation and improvement}

Genomic location information for introns annotated in An. funestus gene set AfunF1.2 and the mitochondrial genome was extracted from the genome annotation. Similar location information for introns identified by alignment of RNAseq data, for the poly(A) samples, was extracted and the two datasets were compared to identify introns validated by RNAseq. Similar analyses were done for $5^{\prime}$ and 3 ' intron ends alone. For validated introns, sequences spanning the $5^{\prime}$ and $3^{\prime}$ intron ends were extracted and used to identify enriched nucleotide motifs, represented as sequence logos using WebLogo $3[47,48]$.

Manual genome annotation improvement was carried out using Anopheles gambiae gene models as a reference. Starting with translated An. gambiae genes, BLASTp similarity searches were carried out in VectorBase to define the closest matching An. funestus proteins and identify gross differences in protein length. Then, gene models were compared and An. funestus gene models modified to match those of An. gambiae where appropriate. RNAseq alignments were used to guide the An. funestus gene model editing. As transcript ends are difficult to define from RNAseq alignments (due to coverage drop off near to molecule ends), edited gene models included putative protein coding regions but not putative un-translated regions. Edited gene models were submitted to VectorBase [12, 13].

\section{Clustering of sequences from multiple transcriptome datasets and assignment of scaffolds to chromosome arms}

Sequences from two transcriptome assemblies were downloaded from NCBI [49]: the "Crawford" transcriptome, comprising 14,850 sequences (accessions EZ966136-EZ980985) and the "Gregory" transcriptome, comprising 18,103 sequences (accessions EZ915182EZ933284). Sequences from the FUMOZ predicted transcript set AfunF1.2, comprising 13,897 sequences, were downloaded from VectorBase [12, 13]. The three datasets were concatenated and clustered by sequence identity using CD-HIT-EST v4.6 [50], with a clustering threshold of $95 \%$ nucleotide identity.

To tentatively assign An. funestus genome assembly scaffolds to chromosome arms, predicted protein sequences from An. funestus (gene set Afun1.2) and $A n$. gambiae (gene set Agam4.3) were downloaded from VectorBase $[12,13]$. Sequence headers were parsed to retain the scaffold/chromosome information and the two datasets concatenated and clustered by peptide sequence identity using CD-HIT v4.6 [50], with a clustering threshold of $75 \%$ amino acid identity. An. funestus scaffolds 
were assigned to a chromosome arm if they contained at least 5 putative 1:1 An. gambiae orthologues from that chromosome arm. Chromosome arms $2 \mathrm{~L}$ and 3R were switched to account for a whole arm translocation between $2 \mathrm{~L}$ and 3R in An. funestus relative to An. gambiae.

\section{Availability of supporting data}

The RNAseq read data reported in this study were submitted to the European Nucleotide Archive (ENA) under the study accession PRJEB10294 (http://www.ebi.ac.uk/ ena/data/view/PRJEB10294) and the sample accessions ERS809802-ERS809809.

\section{Additional files}

Additional file: 1: Detailed and extra information and figures. File containing detailed descriptions of the analyses and figures not included in the main article. (PDF $3540 \mathrm{~kb}$ )

Additional file 2: Differential gene representation analysis results. Table of results of differential gene representation analysis (for gene set Afun1.2 and mitochondrial genes) contrasting Ribo-Zero and poly(A) enriched RNAseq libraries. The most over-represented genes in Ribo-Zero libraries are highlighted in red. The most over-represented genes in poly(A) libraries are highlighted in blue. The significance cutoff used was an FDR-adjusted (FDR $=5 \%) P$-value $<0.01$. The tag counts for FANG-1 samples are the mean of two libraries (see main text). (XLSX $1784 \mathrm{~kb}$ )

Additional file 3: Validation of annotated exon-exon junctions. Table of exon-exon junctions annotated in gene set Afun1.2 and the mitochondrial genome that were, or were not, validated by RNAseq data generated in this study. Introns validated at both ends (labelled 5'_and_3') or at only one end (labelled 5'_only or 3'_only) are indicated. (XLSX $1949 \mathrm{~kb}$ )

Additional file 4: Annotation improvements. Table of gene annotation validation and/or manual improvement of Anopheles funestus detoxification gene models. Gene models for genes involved in the detoxification of xenobiotics were checked against both Anopheles gambiae orthologues and RNAseq alignments and modified where necessary. (XLSX $40 \mathrm{~kb}$ )

Additional file 5: Integration of transcriptome datasets. Table of sequence clusters (clustered at $95 \%$ sequence identity) comprising AfunF1.2 gene set transcripts (13,897 sequences), the "Crawford" assembled transcriptome (14,850 sequences) and the "Gregory" assembled transcriptome (18,103 sequences). Each row represents a cluster. Sequences in a cluster that come from the same dataset are separated by commas. (XLSX $868 \mathrm{~kb}$ )

Additional file 6: Scaffold assignment to chromosome arms. Table of Anopheles funestus scaffolds assigned to chromosome arms. Assignment is based on the possession of at least 5 putative 1:1 orthologues with Anopheles gambiae occurring on the same chromosome arm. Orthology was inferred by clustering predicted protein sequences at $75 \%$ amino acid identity. (XLSX $43 \mathrm{~kb}$ )

\footnotetext{
Abbreviations

NGS: Next-generation sequencing; PCA: Principal component analysis: DGE: Differential gene expression; TMM: Trimmed mean M-values; LR: Likelihood ratio; FDR: False discovery rate; UTR: Un-translated region; GO: Gene ontology; RZ: Ribo-Zero; PA: Poly(A); P450: Cytochrome P450 monooxygenase; GST: Glutathione S-transferase; COE: Carboxylesterase; FMO: Flavin-containing monooxygenase; AOX: Aldehyde oxidase; ALDH: Aldehyde dehydrogenase; UGT: UDP glucosyltransferase; SULT: Sulfotransferase; ABC: ATP-binding cassette transporter.
}

\section{Competing interests}

The authors declare no competing interests.

\section{Authors' contributions}

GDW and CSW conceived the study. HI carried out RNA extraction and pooling. MH carried out mRNA enrichment and sequence library preparation. GDW carried out bioinformatic analyses and wrote the manuscript. CSW participated in coordination of the study and the writing of the manuscript. All authors read and approved the final manuscript.

\section{Acknowledgements}

We are grateful to Prof. Lizette Koekemoer (University of the Witwatersrand, South Africa) for the kind gift of FANG colony mosquito eggs. Sequence data were generated at the Centre for Genomic Research, which is based at the University of Liverpool. We are grateful to Dr. Yongxiang Fang (Centre for Genomic Research, University of Liverpool) for scripts and advice on the analysis of differential gene expression data. This work was supported by a Wellcome Trust Senior Research Fellowship in Biomedical Sciences (101893/ Z/13/Z) awarded to CSW.

\section{Author details}

VVector Biology Department, Liverpool School of Tropical Medicine, Pembroke Place, Liverpool L3 5QA, UK. ${ }^{2}$ Centre for Genomic Research Institute of Integrative Biology, University of Liverpool, Crown Street, Liverpool L69 7ZB, UK.

Received: 2 September 2015 Accepted: 20 October 2015

Published online: 14 November 2015

\section{References}

1. Sinka ME, Bangs MJ, Manguin S, Coetzee M, Mbogo CM, Hemingway J, et al. The dominant Anopheles vectors of human malaria in Africa, Europe and the Middle East: occurrence data, distribution maps and bionomic précis. Parasites Vectors. 2010;3:117.

2. Morgan JC, Irving H, Okedi LM, Steven A, Wondji CS. Pyrethroid resistance in an Anopheles funestus population from Uganda. PLoS One. 2010;5:e11872.

3. Wondji CS, Coleman M, Kleinschmidt I, Mzilahowa T, Irving H, Ndula M, et al. Impact of pyrethroid resistance on operational malaria control in Malawi. Proc Natl Acad Sci U S A. 2012;109:19063-70.

4. Riveron JM, Irving $H$, Ndula M, Barnes KG, Ibrahim SS, Paine MJ, et al, Directionally selected cytochrome P450 alleles are driving the spread of pyrethroid resistance in the major malaria vector Anopheles funestus. Proc Natl Acad Sci U S A. 2013;110:252-7.

5. Riveron JM, Yunta C, Ibrahim SS, Djouaka R, Irving H, Menze BD, et al. A single mutation in the GSTe2 gene allows tracking of metabolically based insecticide resistance in a major malaria vector. Genome Biol. 2014;15:R27.

6. Riveron JM, Ibrahim SS, Chanda E, Mzilahowa T, Cuamba N, Irving H, et al. The highly polymorphic CYP6M7 cytochrome P450 gene partners with the directionally selected CYP6P9a and CYP6P9b genes to expand the pyrethroid resistance front in the malaria vector Anopheles funestus in Africa. BMC Genomics. 2014;15:817.

7. Choi KS, Christian R, Nardini L, Wood OR, Agubuzo E, Muleba M, et al. Insecticide resistance and role in malaria transmission of Anopheles funestus populations from Zambia and Zimbabwe. Parasites Vectors. 2014;7:464.

8. Mulamba C, Riveron JM, Ibrahim SS, Irving H, Barnes KG, Mukwaya LG, et al. Widespread pyrethroid and DDT resistance in the major malaria vector Anopheles funestus in East Africa is driven by metabolic resistance mechanisms. PLoS One. 2014;9:e110058.

9. Glunt KD, Abílio AP, Bassat Q, Bulo H, Gilbert AE, Huijben S, et al. Long-lasting insecticidal nets no longer effectively kill the highly resistant Anopheles funestus of southern Mozambique. Malar J. 2015;14:298.

10. Holt RA, Subramanian GM, Halpern A, Sutton GG, Charlab R, Nusskern DR, et al. The genome sequence of the malaria mosquito Anopheles gambiae. Science. 2002;298:129-49

11. Neafsey DE, Waterhouse RM, Abai MR, Aganezov SS, Alekseyev MA, Allen JE, et al. Mosquito genomics. Highly evolvable malaria vectors: the genomes of 16 Anopheles mosquitoes. Science. 2015;347:1258522.

12. Giraldo-Calderón Gl, Emrich SJ, MacCallum RM, Maslen G, Dialynas E, Topalis $\mathrm{P}$, et al. VectorBase: an updated bioinformatics resource for invertebrate vectors and other organisms related with human diseases. Nucleic Acids Res. 2015:43(Database issue):D707-13.

13. VectorBase. https://www.vectorbase.org 
14. Cantarel BL, Korf I, Robb SM, Parra G, Ross E, Moore B, et al. MAKER: an easy-touse annotation pipeline designed for emerging model organism genomes. Genome Res. 2008;18:188-96.

15. Holt C, Yandell M. MAKER2: an annotation pipeline and genome-database management tool for second-generation genome projects. BMC Bioinformatics. 2011;12:491

16. Djebali S, Davis CA, Merkel A, Dobin A, Lassmann T, Mortazavi A, et al. Landscape of transcription in human cells. Nature. 2012;489:101-8.

17. de Klerk E, 't Hoen PA. Alternative mRNA transcription, processing, and translation: insights from RNA sequencing. Trends Genet. 2015;31:128-39.

18. Cui $P$, Lin Q, Ding F, Xin C, Gong W, Zhang L, et al. A comparison between ribo-minus RNA-sequencing and polyA-selected RNA-sequencing. Genomics. 2010;96:259-65.

19. Yang L, Duff MO, Graveley BR, Carmichael GG, Chen LL. Genomewide characterization of non-polyadenylated RNAs. Genome Biol. 2011;12:R16.

20. Zhao W, He X, Hoadley KA, Parker JS, Hayes DN, Perou CM. Comparison of RNA-Seq by poly (A) capture, ribosomal RNA depletion, and DNA microarray for expression profiling. BMC Genomics. 2014;15:419.

21. Sultan M, Amstislavskiy V, Risch T, Schuette M, Dökel S, Ralser M, et al. Influence of RNA extraction methods and library selection schemes on RNA-seq data. BMC Genomics. 2014;15:675.

22. Crawford JE, Guelbeogo WM, Sanou A, Traoré A, Vernick KD, Sagnon N, et al. De novo transcriptome sequencing in Anopheles funestus using Illumina RNAseq technology. PLoS One. 2010;5:e14202.

23. Gregory R, Darby AC, Irving H, Coulibaly MB, Hughes M, Koekemoer LL, et al. A de novo expression profiling of Anopheles funestus, malaria vector in Africa, using 454 pyrosequencing. PLoS One. 2011;6:e17418.

24. Winnebeck EC, Millar CD, Warman GR. Why does insect RNA look degraded? J Insect Sci. 2010;10:159.

25. Marzluff WF, Wagner EJ, Duronio RJ. Metabolism and regulation of canonical histone mRNAs: life without a poly(A) tail. Nat Rev Genet. 2008;9:843-54.

26. Kühl M, Wedlich D. XB/U-cadherin mRNA contains cytoplasmic polyadenylation elements and is polyadenylated during oocyte maturation in Xenopus laevis. Biochim Biophys Acta. 1995;1262:95-8.

27. Beilharz TH, Preiss T. Widespread use of poly(A) tail length control to accentuate expression of the yeast transcriptome. RNA. 2007;13:982-97.

28. Hunt RH, Brooke BD, Pillay C, Koekemoer LL, Coetzee M. Laboratory selection for and characteristics of pyrethroid resistance in the malaria vector Anopheles funestus. Med Vet Entomol. 2005;19:271-5.

29. Martin M. Cutadapt removes adapter sequences from high-throughput sequencing reads. EMBnet J. 2011;17:10-2.

30. Joshi NA, Fass JN. Sickle. A sliding-window, adaptive, quality-based trimming tool for FastQ files (Version 1.33). https://github.com/najoshi/sickle. 2011

31. Krzywinski J, Grushko OG, Besansky NJ. Analysis of the complete mitochondrial DNA from Anopheles funestus: an improved dipteran mitochondrial genome annotation and a temporal dimension of mosquito evolution. Mol Phylogenet Evol. 2006:39:417-23.

32. Kim D, Pertea G, Trapnell C, Pimentel H, Kelley R, Salzberg SL. TopHat2: accurate alignment of transcriptomes in the presence of insertions, deletions and gene fusions. Genome Biol. 2013;14:R36.

33. Langmead B, Salzberg SL. Fast gapped-read alignment with Bowtie 2. Nat Methods. 2012;9:357-9.

34. HTSeq. http://www-huber.embl.de/users/anders/HTSeq/doc/count.html

35. Robinson MD, McCarthy DJ, Smyth GK. edgeR: a Bioconductor package for differential expression analysis of digital gene expression data. Bioinformatics. 2010;26:139-40.

36. Robinson MD, Oshlack A. A scaling normalization method for differential expression analysis of RNA-seq data. Genome Biol. 2010;11:R25.

37. Robinson MD, Smyth GK. Moderated statistical tests for assessing differences in tag abundance. Bioinformatics. 2007;23:2881-7.

38. Nelder JA, Wedderburn RWN. Generalized linear models. J R Stat Soc Ser A 1972;135:370-84.

39. Wilks SS. The large-sample distribution of the likelihood ratio for testing composite hypotheses. Ann Math Stat. 1938:9:60-2.

40. Benjamini $Y$, Hochberg Y. Controlling the false discovery rate: a practical and powerful approach to multiple testing. J R Stat Soc Ser B. 1995;57:289-300.

41. BlastKOALA. http://www.kegg.jp/blastkoala. Accessed April, 2015.

42. Kanehisa M, Goto S. KEGG: kyoto encyclopedia of genes and genomes. Nucleic Acids Res. 2000;28:27-30
43. Kanehisa M, Goto S, Sato Y, Kawashima M, Furumichi M, Tanabe M. Data, information, knowledge and principle: back to metabolism in KEGG. Nucleic Acids Res. 2014;42(Database issue):D199-205.

44. Kyoto Encyclopedia of Genes and Genomes (KEGG) http://www.genome.jp/kegg. Accessed April, 2015.

45. Trapnell C, Williams BA, Pertea G, Mortazavi A, Kwan G, van Baren MJ, et al. Transcript assembly and quantification by RNA-Seq reveals unannotated transcripts and isoform switching during cell differentiation. Nat Biotechnol. 2010;28:511-5.

46. Liao Y, Smyth GK, Shi W. featureCounts: an efficient general purpose program for assigning sequence reads to genomic features. Bioinformatics. 2014;30:923-30.

47. Schneider TD, Stephens RM. Sequence logos: a new way to display consensus sequences. Nucleic Acids Res. 1990:18:6097-100.

48. Crooks GE, Hon G, Chandonia JM, Brenner SE. WebLogo: a sequence logo generator. Genome Res. 2004;14:1188-90.

49. NCBI. http://www.ncbi.nlm.nih.gov

50. Fu L, Niu B, Zhu Z, Wu S, Li W. CD-HIT: accelerated for clustering the nextgeneration sequencing data. Bioinformatics. 2012;28:3150-2.

\section{Submit your next manuscript to BioMed Central and take full advantage of:}

- Convenient online submission

- Thorough peer review

- No space constraints or color figure charges

- Immediate publication on acceptance

- Inclusion in PubMed, CAS, Scopus and Google Scholar

- Research which is freely available for redistribution 